ANUARIO DE EsTUdios MEDIEVALES 48/2, julio-diciembre de 2018, pp. 615-638

ISSN 0066-5061

https://doi.org/10.3989/aem.2018.48.2.03

\title{
POUR EN FINIR AVEC LES CALCURINI
}

\section{PUTTING AN END TO THE ENIGMA OF THE CALCURINI}

\author{
PIERRE-VINCENT CLAVERIE \\ Centre de recherche scientifique de Chypre \\ http://orcid.org/0000-0002-1451-6572
}

\begin{abstract}
Résumé: Depuis cinq siècles, les historiens débattent de l'identité des Calcurini qui tentèrent de subjuguer le port de Ceuta en 1234. Le présent article tente de faire la lumière sur cette énigme, en confrontant les hypothèses formulées à propos des Calcurini évoqués par les sources génoises. Une analyse critique permet de repousser les conclusions des arabisants et de confirmer l'origine catalane assignée naguère aux Calcurini par Ch.-E. Dufourcq. Tout pousse à voir dans ces marins expérimentés des matelots colliourencs aux ordres du seigneur de Roussillon, Nunó Sanç. Une série de traités signés par l'intéressé avec la République de Gênes explique le comportement chevaleresque des Calcurini lors de leur rencontre avec une escadre génoise devant Ceuta en août 1234.
\end{abstract}

Mots clés: Calcurini; Ceuta; Gênes; Nunó Sanç; Collioure.

Abstract: Since the Renaissance, historians have been unable to identify the Calcurini who tried to conquer the port of Ceuta in 1234. The aim of this article is to study the various assumptions put forward about the Calcurini, who appear in the Genoese sources of the thirteenth century. Although many scholars have considered the Calcurini to be Basque, Portuguese or Andalusian, an in-depth analysis establishes their Catalan origins in agreement with an earlier suggestion made by Ch.-E. Dufourcq. These sailors were natives of Collioure employed by the lord of Roussillon, Nunó Sanç, who had signed a series of treaties with Genoa in 1233. This diplomatic background explains the chivalrous behaviour of the Calcurini during their confrontation with a Genoese fleet in the Strait of Gibraltar in August 1234.

Keywords: Calcurini; Ceuta; Genoa; Nunó Sanç; Collioure.

\section{SOMMAIRE}

1. Introduction.- 2. Le témoignage des sources médiévales.- 3. Les thèses soutenues à propos de l'origine des Calcurini.- 4. La solution colliourenque.- 5. Appendice.6. Bibliographie citée.

\section{INTRODUCTION ${ }^{1}$}

La République génoise a affronté dans son histoire nombre de puissances régionales décidées à contrer son essor maritime et à capter ses zones d'influence dans le bassin méditerranéen. Malgré des revers spectaculaires,

\footnotetext{
${ }^{1}$ Cet article développe les conclusions que nous avons exposées lors d'un colloque organisé à Ceuta en octobre 2015.

Cómo citar este artículo: Claverie, Pierre-Vincent (2018), Pour en finir avec les Calcurini, "Anuario de Estudios Medievales" 48/2, pp. 615-638. https://doi.org/10.3989/aem.2018.48.2.03

Copyright: (c) 2018 CSIC. This is an open-access article distributed under the terms of the Creative Commons Attribution 4.0 International (CC BY 4.0) License.
} 
elle sut mettre au pas les cités de Pise et de Venise lors des batailles navales de la Meloria et de Curzola (Korčula) en 1284 et 1298. Une lutte d'influence l'opposa, un demi-siècle plus tôt, à un mystérieux peuple que les sources génoises désignent sous le terme de Calcurini ou Calculini. Les hypothèses les plus fantaisistes ont fleuri afin d'expliquer l'origine de ces combattants engagés dans la conquête du port de Ceuta en $1234^{2}$. La cité marocaine venait alors de faire sécession de l'Empire almohade qui traversait une période de guerres intestines après la défaite de Las Navas de Tolosa. En l'absence de témoignages extérieurs, les historiens se sont crus autorisés à élaborer des théories audacieuses sur la provenance et les motivations des Calcurini, que les annales génoises présentent comme des croisés d'origine ibérique.L'énigme restant entière au terme de cinq siècles de débat, il nous a paru intéressant de rouvrir le dossier et d'avancer une explication fondée sur l'étymologie et l'histoire de la Méditerranée occidentale. Avant d'évaluer les hypothèses formulées depuis la Renaissance, il convient d'étudier le témoignage des sources à partir desquelles les historiens ont glosé. Nous verrons de la sorte que les Calcurini n'étaient nullement des croisés portugais ou des mercenaires chrétiens aux ordres des Almohades, comme des auteurs contemporains le prétendent ${ }^{3}$.

\section{LE TÉMOIGNAGE DES SOURCES MÉDIÉVALES}

C'est au mémorialiste de la Commune de Gênes, Bartolomeo, que l'on doit selon toute vraisemblance la description des faits de guerre des Calcurini. Un lettré de ce nom exerça en effet l'office de scribe communal entre 1225 et 1248, quoiqu'on ait pu avancer l'hypothèse d'un travail collégial pour les treize premières années de cette période sur la base d'éléments paléographiques. Il paraît indispensable de traduire le passage des annales qui relate l'affrontement des Calcurini et des Génois dans le détroit de Gibraltar pour saisir les enjeux de l'affaire. Ceuta représentait la porte d'entrée des Génois dans le Gharb depuis 1160 , au delà de sa position stratégique à l'orée de la mer d'Alboran. Sa seule rivale à l'échelle du Maghreb fut au tournant des XII ${ }^{e}$ et XIII ${ }^{e}$ siècles le port de Bougie qui offrait un itinéraire direct aux Ligures à partir de la Riviera de par sa longitude ${ }^{4}$.

2 Balard 1978; Jehel 1993; Mosquera Merino 1994, pp. 107-111.

${ }^{3}$ Dotson 2006, p. 69; Jehel 2015, p. 65.

${ }^{4}$ Imperiale di Sant'Angelo 1923, pp. X-XVI; Bautier 1953-1954, pp. 402-407; Valérian 2006, pp. 247-330. La conjoncture favorisa à partir des années 1230 le port de Tunis qui multiplia les traités de commerce avec les républiques italiennes. 
De fait durant la même année [1234], à l'occasion de la fête de l'apôtre Barthélemy, la nouvelle parvint de la région de Ceuta que les croisés [nommés] Calcurini étaient venus mettre le siège devant Ceuta avec une très grande armée afin de s'emparer du lieu et de ses habitants (homines). Les Génois, qui étaient dans ses parages avec plusieurs nefs et une grande quantité de besants, de marchandises et de biens, furent saisis de peur. Ils redoutaient en effet la perte de leurs personnes et des biens, si la terre en question venait à être prise par les susdits Calcurini. Ils craignaient également de se battre contre des chrétiens frappés du signe de la Croix. Néanmoins, vu que les Calcurini avaient déjà capturé à Cadix et dans le détroit [de Gibraltar] quelques nefs génoises et plusieurs ressortissants de Gênes comme Guglielmo Negrone et Balduino Spiono, les Génois qui étaient à Ceuta armèrent dix de leurs plus grandes et meilleures nefs afin d'aller contre eux. Mais les Calcurini proférèrent de bonnes paroles et élargirent ledit Guglielmo Negrone et les autres Génois qu'ils détenaient ${ }^{5}$.

Les Génois se retrouvèrent ainsi d'un côté et les Calcurini de l'autre, au vent. Ils mirent le feu à une petite nef vétuste, qui les accompagnait en tant que prise de guerre, et l'envoyèrent en direction des nefs et des lins des Génois afin qu'ils s'embrasassent à son contact. Les nôtres voyant qu'ils ne pouvaient se garder du feu, dispersèrent les affaires et les biens qu'ils avaient à Ceuta et rallièrent Malaga avec les dix nefs en question. Les Calcurini prirent une certaine nef, ou busse, qui avaient été chargée et équipée afin de venir à Gênes. Ils brûlèrent également d'autres lins qui se trouvaient dans les parages. Les Génois des dix nefs repliées à Malaga organisèrent alors une délibération. Il ressortit de ce conseil et de la volonté commune d'armer deux nefs afin d'envoyer à Ceuta 600 combattants qui pourraient assurer la défense de son territoire et de ses biens ; lesquelles nefs gagnèrent Gomera à l'endroit baptisé le Cap Tétouan ${ }^{6}$ afin que les hommes pussent rejoindre Ceuta par la terre ${ }^{7}$.

Alors quatre des dix nefs [de Malaga] rallièrent Gênes, pendant que les autres rejoignaient Tunis avec les deux [nefs] susdites. Le podestat de Gênes, messire Remedio [Rusca de Côme], ayant reçu un appel au secours du sultan de Ceuta lui promettant de contribuer à la moité des dépenses causées par une intervention et même plus à la réquisition du podestat et de la Commune de Gênes, envoya vers lui dix-huit galères avec les quatre nefs qui avaient rallié Gênes et qui étaient censées porter les victuailles

\footnotetext{
${ }^{5}$ Imperiale di Sant'Angelo 1923, p. 72.

${ }^{6}$ Ibidem, p. 73 : "Tarffonoli” qui renvoie au Cap Tétouan, situé à 30 kilomètres au sud de Ceuta.

${ }^{7}$ Imperiale di Sant'Angelo 1923, p. 73.
} 
et les biens nécessaires auxdites galères et nefs. Les premières galées à partir furent les quatre galères dont messire Lanfranco Spinola avait été constitué capitaine. Ensuite, vinrent les dix galères dont le noble Ottobono di Camilla avait été nommé capitaine. Enfin, prirent le large les quatre autres galères et les susdites quatre nefs [de Ceuta] pour lesquelles avait été désigné comme capitaine Ingone di Bonifacio della Volta. Afin de couvrir les dépenses des susdites nefs et galères, on aliéna douze deniers sur dix ans dans chaque mine de sel [génoise] pour un montant de 18.000 livres ${ }^{8}$.

Comme lesdits amiraux ou capitaines exigèrent et requirent du sultan de Ceuta les dépenses qu'il avait promises et le remboursement des dommages passés qui leur avaient été infligés par les Calcurini, ledit sultan craignant la puissance des Génois s'en remit à des Berbères (misit per barbaros). Et comme presque toute la cité de Ceuta était pleine de Berbères, certains des matelots des galères en vinrent aux mains avec plusieurs Sarrasins ; par quoi tous en appelèrent aux armes. Un début de combat eut lieu et les Sarrasins fondirent sur les fondouks pour les incendier avec les maisons attenantes. Plusieurs personnes trouvèrent la mort ainsi ou furent tuées, et une très grande quantité de biens génois fut brûlée et perdue ${ }^{9}$.

Le témoignage des annales génoises est corroboré par une chronique marocaine qui signale le recours à des nomades par l'émir Abū 1-'Abbās al-Yanaštī afin de se défaire des Ligures qui menaçaient la sécurité de Ceuta. Le Bayān al-Mughrib du Marrakéchois Ibn 'Idhārīn'évoque malheureusement pas le contexte qui présida à l'arrivée massive de Génois dans la péninsule tingitane en 1234. Il se contente de signaler leur présence au niveau de la douane et du faubourg de Ceuta pour des raisons commerciales ${ }^{10}$. Le chroniqueur mérinide, Ibn Abī Zar', ne se montre guère plus loquace sur l'épisode, en mentionnant un siège prolongé de Ceuta par les Génois au tournant des années 632-633 de l'Hégire (1234-1236 AD). L'émir al-Yanaštī aurait mis un terme à cette menace, en acceptant de verser aux Ligures un tribut faramineux de 400.000 dinars. Le Bayān al-Mughrib pare du qualificatif de hājj̄̄ l'émir de Ceuta qui avait réalisé un pèlerinage à La Mecque, avant de prendre le contrôle de la ville en 1232. Al-Yanaštī avait profité de l'appui de l'oligarchie marchande de Ceuta pour chasser l'amiral Abū 1-Aṣbag al-Ğuštī qui administrait la ville pour le compte du roi de Murcie, Ibn Hūd, à la suite

\footnotetext{
${ }^{8}$ Ibidem.

${ }^{9}$ Ibidem, pp. 73-74.

${ }^{10}$ Amari 1867, n IV pp. 615-617; 1873, n IV pp. $67-69$ (tiré-à-part); Bresc, et al. 2001, pp. 114-115.
} 
d'une rébellion contre les Almohades. Il parvint à se maintenir au pouvoir jusqu'en 1238, en arborant le titre de "sultan de Ceuta" dans ses rapports avec les chrétiens ${ }^{11}$.

Les archives génoises conservent une série de documents relatifs au coup de main des Calcurini contre Ceuta. Le premier d'entre eux est un engagement solennel en date du 6 septembre 1234, consenti par le Génois Simone Pellicciaio en faveur de son compatriote Pagano Sumaulla. Simone s'oblige à servir en lieu et place de Pagano à bord d'une des quatre galères dépêchées par la Commune à Ceuta contre un salaire de trois livres et seize sous de Gênes. Devant le même notaire Gianuino da Predono, comparaît le lendemain le tisserand Gandolfo da Fontanabuona qui doit embarquer sur la galère du port de Recco, confiée au capitaine Guido Pollicino. Gandolfo reconnaît avoir reçu de Giribaldo da Recco la somme de trois livres et quinze sous de Gênes pour le remplacer à bord du navire en partance pour Ceuta. Ce contrat révèle l'association de plusieurs ports de la Riviera à l'expédition montée contre les Calcurini. Cette mobilisation générale se retrouvera, l'année suivante, dans la mahone organisée afin de prendre le contrôle de Ceuta au nom de la République $^{12}$.

Une reconnaissance de dette de 1237 mentionne, pour sa part, le nom d'une nef brûlée par les Calcurini au large du Gharb. Il s'agit du "San Marco" dont les quatorze seizièmes avaient été achetés par deux habitants de Savone auprès des Génois Bulgaro da Platealonga, Oberto della Porta et Guglielmo da Quarto, sans que l'argent n'ait le temps d'être versé. Aussi Iacopo Caracapa et Guglielmo Formica s'engagèrent-ils à rembourser 547 besants de millarès aux anciens propriétaires du navire, en les associant aux procédures, raisons et actions, tant réelles que personnelles et utiles, dirigées vers le roi, les Sarrasins et la communauté de Ceuta. Leur reconnaissance de dette révèle la nomination de collecteurs afin d'obtenir des Sebtis les sommes devant revenir aux citoyens et à la Commune de Gênes à titre d'indemnités (pro restauratione dampni et perdite). L'emploi du futur dans cet acte de février 1237 montre que l'indemnisation des Génois fut longue et périlleuse malgré la familiarité de l'émir al-Yanaštī avec les questions financières. Les anciens propriétaires du "San Marco" acceptèrent les termes de la transaction, sans imaginer que le "roi de Ceuta" allait perdre son trône de manière anticipée en 1238 dans un contexte d'épidémie et de disette. La dédition de Ceuta au calife almohade, 'Abd al-Wāḥid II, fut l'ultime avatar d'une crise amorcée avec le siège de la cité

${ }_{11}$ Beaumier 1860, p. 394; Huici Miranda 1964, vol. II, pp. 528-529; Ferhat 1993, pp. 213-219.

${ }^{12}$ Tucci 1935 , n 1-2 pp. 282-284. 
par les Calcurini en 1234. Reste à identifier l'origine de ces mystérieux croisés européens ${ }^{13}$.

\section{LES THÈSES SOUTENUES À PROPOS DE L'ORIGINE DES CALCURINI}

C'est à la Renaissance qu'apparaissent les premières explications du gentilé Calcurini sous la plume de chroniqueurs incapables de décrypter les textes médiévaux. Oberto Foglietta dans son Histoire de la République de Gênes fait des Calcurini un peuple de Biscaye (nonnulli ex Cantabris populis), désireux de conquérir Ceuta pour des motifs aussi économiques que religieux dans la mesure où toute guerre contre les ennemis de la foi chrétienne est légitime. Il leur dénie toute volonté de conciliation avec les Génois, la libération des prisonniers pris devant Cadix n'étant à ses yeux qu'un subterfuge voué à faciliter la conquête de Ceuta par les croisés (amicum ac pacatum animum simulantes $)^{14}$. Son compatriote Giustiniani se veut plus objectif dans le récit de l'expédition des Calcurini qu'il donne en 1537 dans ses Annales expurgées de la République de Gênes. Il suit à la lettre le récit de Bartolomeo Scriba, en attribuant aux Calcurini des origines galiciennes, basques ou navarraises que les historiens italiens ont érigés en certitude au fil des siècles suivants ${ }^{15}$.

Le premier auteur à se démarquer de cette vision fut Louis de Mas Latrie dans un recueil diplomatique présentant les Calcurini comme une émanation des "ordres militaires d'Espagne". W. Heyd relaya cette lecture dans un essai sur les comptoirs italiens au moment où M. Amari publiait un corpus de documents arabes éclairant l'histoire génoise. Le savant arabisant avança dans l'introduction de ce travail l'hypothèse d'une instrumentalisation des Calcurini par le calife almohade, 'Abd al-Wāhid II, afin de prendre le contrôle de Ceuta. Il accusa Bartolomeo Scriba de s'être mépris sur la chronologie des événements et les croix arborées par les Calcurini sur leurs vaisseaux. Les adversaires des Génois ne pouvaient être, selon lui, que des éléments de la milice chrétienne que le calife almohade, al-Ma'mūn, avait dépéchée contre Ceuta lors de son soulèvement en 1232. Amari profita de l'occasion pour rejeter l'identification des Calcurini avec des combattants de Calahorra qui

${ }^{13}$ Ibidem, n 31-32 pp. 338-339. Le millarès était une monnaie d'argent à neuf ou dix douzièmes de fin que les chrétiens exportaient massivement vers le Maghreb au XIII ${ }^{\mathrm{e}}$ siècle en raison de son taux de change avantageux avec l'or.

${ }^{14}$ Foglietta 1585, f. 64v.

${ }^{15}$ Giustiniani 1537, liv. 3, G-1234; Deza 1694, pp. 101-102; Canale 1844, vol. II, p. 84; Promis 1865, p. 15, note **; Balan 1873, vol. II, pp. 326-327; Caddeo, Nani-Mocenigo 1942, vol. I, p. 393; Unali 2000 , pp. 78-79. 
se seraient retrouvés, on ne sait comment, à la tête d'une flotte de guerre au large du Maroc ${ }^{16}$.

Sa théorie fut bien accueillie par la communauté scientifique malgré le discrédit apporté aux annales génoises ${ }^{17}$. C'est l'une des hypothèses les plus communément reprises par les historiens contemporains, bien que Ch.-E. Dufourcq ait révélé ses points faibles en 1955. Le Français établit que les Almohades n'avaient pas de flotte en 1234 et que leur armée opérait à des lieux de la péninsule tingitane quand Ceuta subit l'attaque des Calcurini. On ajoutera à ces remarques que les informateurs de Bartolomeo Scriba étaient parfaitement capables de distinguer une armada croisée des mercenaires chrétiens à la solde des Almohades, si lui-même ne pouvait le faire objectivement. Le mode de consignation des annales génoises interdit en outre toute erreur de millésime à l'égard du raid mené contre Ceuta à l'été 1234. Un raisonnement analogue conduisit Dufourcq à réfuter l'hypothèse d'une appartenance des Calcurini à un ordre militaire ibérique. Aucune source ne signale en effet l'armement d'une flotte de guerre par les ordres de Calatrava, Alcántara ou Santiago au cours de l'année 1234. Quant à la marine castillane, il faudra attendre la conquête de Séville en 1248 pour la voir en position de menacer le Gharb. Dufourcq se montra moins expéditif à l'égard d'une possible origine portugaise des Calcurini. Il évoqua l'organisation de raids antérieurs contre Ceuta, avant de souligner l'investissement des Portugais dans la conquête de l'Algarve au début des années $1230^{18}$.

Ces objections l'amenèrent à assigner une origine catalane aux Calcurini sur la base d'un faisceau convergent d'indices. Le médiéviste français rapprocha le raid sur Ceuta de la conquête d'Ibiza et de Formentera accomplie par l'archevêque de Tarragone, Guillem de Montgrí, en 1235. Cette expédition auréolée du statut de croisade aurait pu être devancée par une attaque sur Ceuta dans la foulée de la prise du port de Peníscola en 1233. Dufourcq souligna l'expérience maritime du seigneur de Roussillon, Nunó Sanç, qui prit part à la conquête d'Ibiza, ainsi que le bellicisme de l'infant Pierre de Portugal qui administrait les Baléares pour le compte du roi d'Aragon depuis 1231. Il invoqua en faveur de son hypothèse un passage de la Grande Chronique de Matthieu Paris, faisant état de l'attaque d'une coalition génoise, pisane et aragonaise sur Ceuta au cours de l'année 1236. Ce texte prétend que les Sebtis

\footnotetext{
${ }^{16}$ Mas Latrie 1866, part. I, p. 81; Heyd 1868, vol. II, pp. 380-382; Amari 1867, pp. 549-748; 1873, pp. 3-144.

${ }_{17}$ Belgrano 1874, p. 487; Imperiale di Sant'Angelo 1923, note 3 p. 72; Di Tucci 1935, p. 279; Ferhat 1993, p. 216; Jehel 2015, p. 65.

${ }^{18}$ Dufourcq 1955, pp. 89-91.
} 
auraient promis de remettre la ville aux chrétiens, en l'absence de secours almohade dans un délai de trois ans. Dufourcq interpréta cette fable comme la preuve que les Calcurini étaient des ressortissants de la Couronne d'Aragon. Restait à expliquer leur présence dans l'Atlantique avant le déclenchement du blocus naval de Ceuta. Le Français avança une confusion possible entre les villes d'Ibiza et de Cadix dans le récit des événements donné par Bartolomeo Scriba. Il reconnut toutefois la légèreté de cette hypothèse et la part de mystère attaché à la présence des Catalans à l'ouest du détroit de Gibraltar en $1234^{19}$. Les travaux ultérieurs de Dufourcq reprirent ces conclusions, sans apporter la preuve formelle de l'origine catalane des Calcurini ${ }^{20}$.

L'historiographie contemporaine intégra l'hypothèse de Dufourcq parmi les plus probables avec les interprétations de Giustiniani et d'Amari qui avaient gagné des partisans au fil des ans. Georges Jehel tenta de concilier ces points de vue en considérant les Calcurini comme des agents du pouvoir barcelonais, chargés d'enrayer la progression des Génois au Maghreb. Cette mission aurait pris la forme d'un recrutement temporaire par le calife almohade, 'Abd al-Wāhid II, afin de rentrer en possession de Ceuta ${ }^{21}$. Plusieurs auteurs se refusèrent à trancher entre les théories existantes, en l'absence d'éléments tangibles dans la documentation du XIII ${ }^{\mathrm{e}}$ siècle. En 1968, H.L. Misbach rompit avec cette prudence en faisant des Calcurini un contingent hudide de Murcie, qui aurait affiché des croix sur ses navires afin de plonger dans l'effroi les Sebtis. On a peine à concevoir l'avantage que les Andalous auraient pu tirer de ce subterfuge en raison de la résistance antérieure de Ceuta à plusieurs assauts chrétiens. Un historien marocain ne craignit pas de remettre en cause l'existence même des Calcurini, en qualifiant de "récits incertains" le compte-rendu de Bartolomeo Scriba. Les réserves de Germain Ayache ne sont pas sans rappeler l'approche hypercritique développée en 1867 par Amari à l'égard du récit des annales génoises $^{22}$. Il ne faut pas s'étonner, dès lors, de voir certains auteurs prendre les Calcurini pour un contingent de Génois alliés de l'émir al-Yanaštī durant sa dissidence ! Un article récent n'hésite pas à dater, pour sa part, du 24 août 1234 leur attaque qui intervint plusieurs jours auparavant. Christophe Picard assigne de son côté le millésime 1231 à l'expédition des Calcurini dans un essai qui reconnait leur puissance navale au même titre que leur origine catalane ${ }^{23}$.

${ }^{19}$ Dufourcq 1955, pp. 92-97; Matthieu Paris 1876, pp. 366-367.

${ }^{20}$ Dufourcq 1966, p. 51; 1969, pp. 131-140.

${ }^{21}$ Lalinde Abadía 1979, p. 41; Zaïm 1990, p. 56; Chérif 1996, pp. 35 et 106; Jehel, Racinet 2000, p. 126; Jehel 2001, pp. 62 et 196.

${ }^{22}$ Rosenberger 1995, p. 28; Refass 1996, p. 45; Picard 1997, pp. 174-175; Misbach 1968, p. 94 ; Ayache 1979 , p. 312 .

${ }_{23}$ Jehel 2015, note 63 p. 72; Picard 2015, p. 341. 
On doit à M.C. Mosquera Merino la première remise en cause sérieuse de la théorie de Dufourcq dans sa thèse sur la seigneurie de Ceuta au $\mathrm{XIII}{ }^{\mathrm{e}}$ siècle. L'historienne espagnole invoqua la caution scientifique de G. Pistarino pour montrer que la théorie catalaniste était loin de triompher à l'échelle du monde méditerranéen. Elle démentit en outre la volonté des Catalans de terrasser la flotte sebtie qui aurait pu compromettre la conquête d'Ibiza par l'intensité de ses liens avec les Baléares. Elle estima, par ailleurs, que les Calcurini avaient suivi un itinéraire occidental, avant de paraître devant Cadix et Ceuta en 1234. Des Catalans auraient privilégié a contrario un itinéraire oriental à partir de Barcelone, sans s'aventurer dans l'Atlantique. La chose n'avait pas échappé du reste aux érudits génois de la Renaissance qui avaient conféré une origine cantabrique ou basque aux Calcurini. Devant la faiblesse de la marine castillane au XIII ${ }^{\mathrm{e}}$ siècle, M.C. Mosquera Merino opta pour une probable origine portugaise des Calcurini. Elle mentionna les attaques antérieures des Portugais sur les côtes du Gharb, en reliant une bulle de croisade du 21 octobre 1234 au blocus de Ceuta effectué durant l'été de la même année ${ }^{24}$. L'historienne espagnole avoua dans un article ultérieur ne pas connaître les buts de guerre exacts des Calcurini, qui pouvaient envisager autant de piller la "clé du Gharb" que de la conquérir au nom de la chrétienté. Force est de constater que sa théorie fut impuissante à expliquer l'étymologie du mot $\mathrm{Cal}$ curini, que nous croyons pouvoir relier à un port du Roussillon bien connu ${ }^{25}$.

\section{LA SOLUTION COLLIOURENQUE}

Il peut sembler étonnant qu'aucun chercheur n'ait songé à relier les adversaires des Génois au seul port en eau profonde des Pyrénées-Orientales. La raison incombe à une mauvaise connaissance de la toponymie ainsi que de l'histoire roussillonnaises. Le substantif Caucoliberi a de fortes chances d'avoir donné naissance au gentilé Calcurini à travers une forme hybride en Calcoliberini. Cet emprunt linguistique semble avoir eu lieu à partir du nom latin de Collioure plutôt que de ses avatars romans en vigueur à l'époque féodale. Les formes Cochliure, Cogliure ou Copliure auraient en effet abouti à un gentilé tout autre si elles avaient servi de sources d'inspiration aux Ligures $^{26}$. Tel n'est pas le cas de la forme latine Calcolibero, que certains textes donnent comme synonyme de Caucolibero, en croyant distinguer une racine

${ }^{24}$ Mosquera Merino 1991, vol. I, pp. 137-148; 1994, pp. 105-111; Pistarino 1980, pp. 189-205.

${ }_{25}$ Mosquera Merino 2002, pp. 114-115.

${ }^{26}$ Basseda 1990, § 577 pp. 401-402. 
latine dans le nom de l'ancien port de la cité d'Illibéris (Elne). Il est probable que des oreilles génoises durent le rapprocher du patronyme Calcolino, attesté en Ligurie au XIII ${ }^{e}$ siècle. Le gentilé qui en émergea tomba, quoi qu'il en soit, en désuétude au siècle suivant lorsque le toponyme de Colliveri s'imposa dans la péninsule italienne comme nom officiel de Collioure ${ }^{27}$.

Un cliché voudrait en outre que le port de Collioure ne se soit développé que dans la seconde moitié du XIII' siècle à l'instigation de Jacques le Conquérant. Ce dernier demande en effet dans son testament de 1272 que son fils Jacques II affecte 5.000 sous des revenus annuels de Collioure à l'entretien du port qu'il a entrepris d'aménager devant la capitale de la Côte Vermeille. Le même acte reconnaît l'usage ancestral du havre de Port-Vendres, qui doit subir une campagne de restauration préalable avant l'achèvement des travaux initiés à Collioure ${ }^{28}$. Bien que la leude de Collioure de 1249 soit restée célèbre, un texte de 1317 fait état de son prélèvement continu depuis plus de 150 ans. Cela n'est guère surprenant dans la mesure où les comtes de Roussillon avaient fait de Collioure leur port d'embarquement pour la Terre sainte et une source de revenus appréciable, en percevant une leude de 3.500 sous melgoriens par an. L'intérêt de la place n'échappa pas à Nunó Sanç qui offrit 400 marcs d'argent, en plus du fief de Thuir, en 1218 à l'héritière de Guillem Durfort afin d'en prendre le contrôle avec la vallée de Banyuls et l'ensemble de ses juridictions maritimes et terrestres ${ }^{29}$.

Collioure offrait un mouillage de qualité aux vaisseaux qui faisaient relâche au niveau de ses ports d'amont et d'avall, localisés de part et d'autre du château royal. Ces installations se limitaient au début du XIII ${ }^{e}$ siècle aux plages de l'Anse Gerbal et du raval -ou faubourg-sur lesquelles une trentaine de galères pouvaient être tirées hors de l'eau. Un privilège de 1207 nous apprend que les marins de Collioure avaient l'obligation de participer aux expéditions des rois d'Aragon dans un périmètre de 180 miles nautiques, allant de Barcelone à Montpellier ${ }^{30}$. Ce droit d'ost fut concédé en 1212 au prince catalan, Nunó Sanç, lorsqu'il hérita de la "seigneurie des comtés de Roussillon et de Cerdagne". Cette titulature étrange s'explique par le fait qu'il administrait les comtés nord-catalans au nom de son père Sanç et du roi d'Aragon, Pierre le Catholique, qui régnait sur un agrégat de territoires catalans, occitans et pro-

${ }^{27}$ Doehaerd 1941, vol. III, pp. 408, 409 et 706; Pegolotti 1936, pp. 113, 122, 167 et 220; Dini 1980 , p. 59.

${ }^{28}$ Udina i Abelló 2001, n 21 pp. 144-146; Alart 1881,pp. 51-59.

${ }^{29}$ Vidal 1887, p. 77; Alart 1874, p. 160; Bisson 1989, pp. 376-379. Guillem Durfort avait acquis ces droits du roi Pierre II d'Aragon en remboursement d'une créance de 60.000 sous de Barcelone.

${ }^{30}$ Bonet 1895-1896, p. 169; Alart 1874, p. 89. 
vençaux. Nunó Sanç était son cousin et un homme ambitieux. Il est resté célèbre pour avoir promulgué une paix généralisée dans le Roussillon en 1217 et acquis, douze ans plus tard, la vicomté de Fenouillèdes et le Peyrapertusès. Il ne craignit pas de prêter hommage pour ces fiefs dès 1226 au roi de France, en arborant le titre de comte de Roussillon, Conflent, Vallespir et Cerdagne, que lui refusait la Maison d'Aragon. L'élément maritime semble lui avoir été familier en raison des déplacements fréquents qu'il effectua en Provence dans sa jeunesse afin d'assurer la tutelle du comte Raymond Bérenger V. C'est de toute évidence ce prince catalan qui tenta de conquérir Ceuta en 1234, après avoir signé un traité d'amitié en 1231 avec la Commune de Montpellier ${ }^{31}$.

La puissance navale de Nunó Sanç transparaît du récit de la conquête de Majorque consigné dans les mémoires de Jacques ${ }^{\text {er }}$ d'Aragon. Si l'on en croit le Llibre dels fets, le cousin du roi aurait déclaré en 1228 que la prospérité et la quiétude de ses possessions lui permettaient de participer à la conquête des Baléares avec la flotte de lins et de galères qu'il tenait armée pour ses besoins personnels. Le seigneur de Roussillon monnaya sa participation à l'expédition contre des domaines agricoles et une dotation de 1.874 fiefs de chevalerie. Il devint ainsi, en 1230, le plus gros propriétaire foncier de Majorque après le roi qui s'était arrogé la moitié des 13.450 “chevaleries" instituées dans l'île $e^{32}$. Les archives majorquines livrent les noms de plusieurs membres de la mesnie de Nunó Sanç qui rassemblait une centaine de chevaliers en 1229. On trouve dans ce corps d'élite des Catalans aux côtés d'étrangers comme le Castillan Pelayo Núñez, l'Occitan Bertran d'Aran et le Français Baudouin de Semy qui hérita de biens sur les territoires de Palma et de Felanitx en 1238-1239. Il suffit de parcourir le préambule des chartes d'inféodation de Nunó Sanç des années 1230 pour se convaincre de l'esprit de croisade qui l'animait à la veille de l'attaque de Ceuta:

Qu'il soit manifeste à tous que nous, Nunó Sanç, seigneur de Roussillon, Vallespir, Conflent et Cerdagne par la grâce de Dieu, voulons et désirons peupler notre terre de l'île de Majorque en l'honneur de Dieu et de sa très sainte et insigne Génitrice, et que nous désirons la protéger des mains des ennemis de la sainte Croix et la défendre virilement afin que les nations barbares ne puissent y prévaloir après l'apport du culte divin ${ }^{33}$.

${ }^{31}$ D'Achery 1723, pp. 587-589; Teulet 1866, n 1768 p. 79 et n 1806 pp. 93-94; Germain 1861, vol. I, n X pp. 191-194.

${ }^{32}$ Bruguera 1991, vol. II, chap. 51 pp. 61-62; Santamaría 1990, pp. 346-347; Tréton, Vinas $2017, \S 13$.

${ }^{33}$ Aguiló 1912-1915, t. 14, pp. 251, 256, 274-275, 281 et t. 15, p. 54; Vich y Salom, Muntaner y Bujosa 1945, n 23 pp. 31-32, 17 pp. 23-25, 21 pp. 29-30. Les vassaux de Nunó Sanç recueillirent au total près de $38 \%$ de ses fiefs majorquins. 
Le goût pour l'aventure de Nunó Sanç est attesté par le raid qu'il effectua le long des côtes barbaresques au printemps 1230 avec une nef et deux galères armées à Majorque. Cette opération de course fut si fructueuse qu'un nombre considérable de Majorquins s'investirent, dans les années qui suivirent, dans des armements particuliers ad lucrandum contra Sarracenos. Nunó Sanç élabora, quant à lui, rapidement un projet de conquête de l'île d'Ibiza avec le soutien de l'infant Pierre de Portugal qui avait reçu la seigneurie du royaume de Majorque en échange de ses droits sur le comté d'Urgel, acquis par voie de mariage. Par un privilège en date du 29 septembre 1231, Jacques le Conquérant leur accorda deux ans pour passer à l'acte et tenir en fief de la Couronne les îles de Formentera et d'Ibiza, d'où ils pourraient mener la guerre contre les musulmans et les chrétiens qui leur seraient hostiles ${ }^{34}$. Nunó Sanç s'investit dans la réalisation de ce projet, en séjournant sans interruption à Majorque entre le 28 juillet 1232 et le 10 juillet 1233. Il ne parvint cependant à recruter assez d'hommes pour entamer la conquête d'Ibiza avant l'échéance fatidique de la Saint-Michel 1233. Aussi préféra-t-il hiverner en Catalogne pour remplir ses obligations féodales envers Jacques le Conquérant et planifier de nouvelles opérations de course contre les musulmans. Les archives aragonaises signalent son absence de la Cour royale entre les 23 janvier et 13 octobre 1234. Ce laps de temps lui permit d'armer l'escadre colliourenque qui entreprit le blocus de Ceuta à la faveur de l'été $1234^{35}$.

L'expédition de Nunó Sanç visait à intercepter les navires opérant au large de l'Andalousie et à subjuguer Ceuta, dont Pierre de Portugal avait apprécié la position stratégique lors d'un séjour au Maroc. Le seigneur de Roussillon faisait peu de différence entre les différents pavillons naviguant en Méditerranée occidentale. C'est ainsi que son testament révèle le désir de dédommager plusieurs Pisans victimes de sa rapacité avec l'équipage d'un lin montpelliérain, arraisonné par ses hommes. Nunó Sanç confessa à cette occasion la saisie arbitraire d'une nef pisane faisant relâche dans le port de Barcelone. Ces manières semblent lui avoir attiré l'hostilité des autorités génoises au début des années 1230. Un règlement du 27 mai 1233 rapporte en effet l'arraisonnement au large de la Riviera d'une nef colliourenque répondant au nom de "L'Angelet" 36 . La trêve de 1233 aboutit à la signature d'un traité d'amitié avec l'ambassadeur génois, Oberto della Volta, et à la cession à la Commune

\footnotetext{
${ }^{34}$ Bruguera 1991, vol. II, chap. 92, p. 103; Santamaría 1990, pp. 423-430; Villanueva 1851, n VIII-IX pp. 257-260.

${ }^{35}$ Aguiló 1912-1915, t. 14, pp. 209-224 et 241-251; Miret i Sans 2007, pp. 110-113.

${ }^{36}$ Tréton, Vinas 2017; Puncuh 1996, n 310 pp. 108-113; Bibolini 2000, n 944 pp. 28-30. Les accords de 1233 furent signés par les chevaliers Ramon de Tallet, Gilabert de Cruïlles et Simó de Tiba qui appartenaient à la mesnie de Nunó Sanç.
} 
de Gênes d'un terrain à bâtir dans la portion de Palma de Majorque dévolue à Nunó Sanç. Les deux parties s'étant engagées à se secourir sur terre, mer et rivière, on comprend l'application avec laquelle le seigneur de Roussillon élargit en 1234 les Ligures que sa flotte avait capturés sur le chemin de Ceuta. L'arrivée dans la cité de 600 combattants génois dut le dissuader de pousser plus avant ses projets. Aussi y a-t-il de fortes chances pour que la flotte colliourenque ait levé le siège de Ceuta avant l'arrivée des secours envoyés par la Superbe au mois de septembre. Les deux parties n'en gardèrent aucune rancune dans la mesure où Nunó Sanç confirma en 1235 la concession d'une portion de fief à Ser Oberto della Volta ${ }^{37}$.

Ces éléments rendent justice à l'hypothèse de Ch.-E. Dufourcq à propos d'une origine catalane des Calcurini. Les projets expansionnistes de Nunó Sanç bénéficièrent d'un concours de circonstances favorable malgré son incapacité à s'emparer d'Ibiza dans les délais définis par Jacques le Conquérant. Le 11 juin 1233, le pape Grégoire IX accorda en effet trois ans d'indulgences aux Aragonais et Navarrais qui se porteraient au secours du royaume de Majorque et collaboreraient à la lutte contre les "Maures infidèles" sous la houlette de Pierre de Portugal ${ }^{38}$. Ces croisés durent participer aux opérations de course menées par Nunó Sanç à partir de Collioure et de Majorque à l'été 1234. Il est probable que le comte de Roussillon disposait d'une dizaine de navires au vu des dix-huit galères armées par la Superbe afin de débloquer le port de Ceuta. Les patrons de ces vaisseaux sont à rechercher parmi les prud'hommes de Collioure qui souscrivirent en 1231 le traité de Nunó Sanç avec la Commune de Montpellier. On retrouve, parmi ces treize notables, des membres des familles de Banyuls, Perés, Ros et Ramon qui prospérèrent sous les rois de Majorque. Les compagnons d'armes de Nunó Sanç doivent être recherchés, pour leur part, parmi les 168 membres de sa cour dont les archives majorquines et roussillonnaises conservent le souvenir ${ }^{39}$. L'un des artisans de la campagne dut être le Génois Carrocino di Alammo qui avait perdu son titre d'amiral de la Couronne d'Aragon en 1231 au profit du Catalan Garcia d'Horta. Nous savons que le fondateur de la famille Carròs avait rompu les ponts avec ses compatriotes en 1229 , en menant un raid fructueux contre le port d'Oran ${ }^{40}$.

La perte des minutiers colliourencs du XIII ${ }^{\mathrm{e}}$ siècle et le laconisme de la Chronique de Perpignan nous empêchent de connaître la composi-

\footnotetext{
${ }^{37}$ Aguiló 1912-1915, t. 14, pp. 249 et 274-275.

${ }^{38}$ Pérez 1961-1967, n 6 p. 53.

${ }^{39}$ Infra Appendice. B) Liste des prud'hommes de Collioure en 1231; Tréton, Catafau, Verdon 2011, vol. I, p. CCVII-CCXX et vol. II, pp. 7-197; infra Appendice. A) Liste des membres de la cour de Nunó Sanç en 1231-1233.

${ }^{40}$ Claverie 2013.
} 
tion exacte de la flotte qui couvrit en 1234 le millier de kilomètres séparant Collioure de Ceuta ${ }^{41}$. Des recherches approfondies permettront peut-être d'exhumer des documents en rapport avec cette expédition et de jauger la contribution des Roussillonnais à la conquête d'Ibiza dans la première moitié de l'année 1235. Nous nous contenterons, pour l'heure, de souligner l'origine colliourenque des navires qui longèrent les côtés andalouses en 1234, avant d'assiéger la "clé du Gharb" pendant quelques semaines. Si une bonne part des combattants embarqués étaient castillans et navarrais du fait des origines maternelles de Nunó Sanç, il faut abandonner l'idée que les Calcurini aient pu servir de supplétifs aux Almohades ou de défenseurs des intérêts barcelonais dans la péninsule tingitane. Leur objectif premier visait à exercer des opérations de guérilla contre les navires musulmans naviguant dans la mer d'Alboran et les "faux chrétiens" qui commerçaient avec les "infidèles" au mépris des interdictions formulées lors des conciles de Latran III et Latran IV. Il y a fort à parier qu'une convention avait été signée par Nunó Sanç et Pierre de Portugal pour se partager le butin pris lors de ces opérations de course ainsi que les places que l'escadre colliourenque pourrait conquérir. La chose ne saurait surprendre de la part des deux hommes qui se partagèrent en bonne intelligence en 1241 les droits de viguerie de la cité de Palma de Majorque, en faisant état publiquement de leur cousinage et affection mutuelle ${ }^{42}$.

\section{APPENDICE}

A) Liste des membres de la cour de Nunó Sanç en 1231-1233 ${ }^{43}$

Il est peu probable que les Italiens mentionnés dans cette liste aient participé à la croisade de Ceuta à l'exception de l'ancien amiral de Catalogne, Carròs, qui avait reçu des biens dans l'île de Majorque en $1229^{44}$.

- Adalbert

- Guillem Adalbert

- Joan Adalbert

${ }^{41}$ Moran i Ocerinjauregui 1998, p. 14. Les plus anciens minutiers colliourencs conservés à ce jour remontent au XIV ${ }^{\mathrm{e}}$ siècle.

${ }^{42}$ Doubleday 2001, pp. 77, 195; Vich y Salom, Muntaner y Bujosa 1945, n 26 pp. 34-35. Nunó Sanç descendait des comtes de Lara par sa mère Sancha Núñez, son père étant le comte Sanç I ${ }^{\mathrm{er}}$ de Roussillon et Cerdagne, disparu en 1224.

${ }^{43}$ Aguiló 1912-1915, 14, pp. 209-224 et 241-251; Alart 1874, pp. 132-138; Germain 1861, vol. I, p. 193; Vich y Salom, Muntaner y Bujosa 1945, pp. 16-30; Cateura Bennàsser 1988, pp. 99-114.

${ }^{44}$ Claverie 2013. 
- Frère Peire d'Agen

- Ramon Aicard

- Pere Albenga

- Pere Anselm

- Pere Antolí

- Berenguer d'Arenys

- Guillem Arnal

- Dalmau de l'Avenc

- Guillem de Banyuls

- Alfons de Barrès

- Arnal de Barrès

- Guillem de Balleres

- Pere Barrau

- Ferrer de Berga

- Bernat de Bonany, vice-notaire comtal

- Bonavia le tanneur

- Nicolau Bonet

- Galceran Bossat

- Lanfranco Bottaro

- Bernat Burget

- Bernat Cala

- Arnal Calafat

- Pons Calard

- Pere Canals

- Pere de Cànoes

- Pere Carceller

- Caroccino ou Carròs ${ }^{45}$

- Arnal de la Casa

- Marquès de Castellet

- Ramon Catell

- Ramon Caulaces

- Domingo Coc

- Ramon Collet

- Pere Comte de Salses

- Ramon de Conilles

- Ramon Cortes

- Gilabert III de Cruïlles

- Guillem Delfí

- Pere Desplanes

- Arnal Desplanter

- Bernat Desplanter

- García Díez

${ }^{45}$ Aguiló 1912-1915, 14, p. 249 : "Corichen" qui déforme le nom de l'ancien amiral royal, Caroccino di Alamanno $(† 1275)$. 
- Gotiero Díez ${ }^{46}$

- Bernat Domènec

- Bernat Eimeric

- Bernat d'Espanya

- Pere Fàbrica

- Pere de Ferrals

- Arnal Ferrer

- Pere Ferrer de Vic

- Guillem Folc

- Ramon de Font, lieutenant comtal

- Domènec de Fos

- Guillem Franquet

- Bernat de La Foia

- Esteve Fuster

- Joan de Galiana

- Guillermo Gallego de Medina

- Pere Garriga

- Pere Gasc

- Guillem Gibot ou Bot

- Arnal Golas

- Ermengol Gros

- Ramon Guerra

- Gil del Hospital

- Bernat Jaspert

- Guerau Jaspert

- Maître Joan, notaire comtal

- Guillem Jordà

- Bartomeu de Jossa

- Julià Juhegues ${ }^{47}$

- Lope Ximénez de Leusia

- Pedro López

- Lanfranco Macolta

- Arnal Malagelada

- Ramon de Malloles

- Bernat Mamet

- Lazzaro di Manzasca

- Bernat Marsili

- Mascaró de Ribesaltes

- Bernardo Mañana

- Hug de Mataplana

- Guillem Matfre

${ }^{46}$ Ce vassal détenait à Majorque depuis 1234 une "chevalerie" exempte de service militaire.

${ }^{47}$ Aguiló 1912-1915, 14, p. 247: "Jusica". 
- Na Maurella ${ }^{48}$

- Galicià de Medina

- Miquel de Montanyans

- Bernat de Monter

- Guillem de Montllorenç

- Guillem Morató

- Pere Morecco

- Fernando de Narváez ${ }^{49}$

- Bernardo de Navarra

- Tomás de Navarra

- Pedro Navarrés

- Juan Núñez

- Pelayo Núñez

- Pere de Oleta

- Guillermo Ortiz

- Bernat de Pabia

- Guillem Ramon de Pa

- Ramon Paó

- Guillem Paó

- Martí Pérez

- Joan Petit

- Martín Pérez de Si

- Francesc Picany

- Guillem Picornell

- Galceran de Pinós

- Pere Poc

- Sanç de Pola

- Guillem de Portella

- Galceran del Pou

- Tomàs del Pou

- Guillem de Porreres

- Martí de Puig Calvel

- Garcia Reboster, intendant comtal

- Guillem Reboster, intendant comtal

- Bernat de Robes

- Domenico di Robio

- Guillem de Rocafort

- Maître Rodolf

- Guillem Roig

- Berenguer Ros

- Rotlan Sabater

- Bernat Sabors

\footnotetext{
${ }^{48}$ Il s'agit de la seule femme appelée à cautionner un acte officiel de Nunó Sanç à Majorque.

${ }^{49} \mathrm{Ce}$ viguier d'origine navarraise apparaît dans les chartes catalanes du XIII' siècle avec les cognomina de Norvaix, Narvax ou Narvaitz.
} 
- Domènec Sabors

- Ramon Sabors

- Armando de Sádaba ${ }^{50}$

- Arnaldo de Sádaba

- Arnal Sala

- Bernat de Sales

- Arnal de Salses

- Garcia Salses

- Bernat Salzet

- Ferrer Salzet

- Pere Salzet

- Hug Sanç

- Rodrigo Sanz

- Domingo Sánchez de Navarra

- Guillem de Sant Donat

- Ferrer de Sant Miquel

- Pere Saroca

- Gonzalo Sartre

- Rodrigo Sartre

- Pere Savila

- Baudouin de Semy

- Pasqual Sisa

- Pierre de Soulages

- Bernat Guillem Suriu

- Ramon de Tallet

- Olivier de Termes

- Arnal de Teulís ${ }^{51}$

- Guillem de Teulís ${ }^{52}$

- Simó de Tiba

- Pere de Tortosa

- Joan Traver

- Bernat Valentí

- Jaume de Vallespir

- Ermengol Venecià

- Pons de Vernet

- Guillem de Vic

- Vicenç ou Vicente

- Mascaró de Vilarasa

- Bernat Xoriguer, scribe comtal, puis notaire public

- Bernardo de Zaragoza

${ }^{50} \mathrm{Ce}$ noble aragonais devait fournir à Nunó Sanç depuis 1233 trois chevaliers et trois écuyers en état de combattre sur terre ou mer.

${ }^{51}$ Aguiló 1912-1915, 14, p. 246 : "Tegures" qui renvoie au village roussillonnais de Taulis.

${ }^{52}$ Ibidem, 14, p. 244 : "Tegures" qui renvoie au village roussillonnais de Taulis. 
B) Liste des prud'hommes de Collioure en $1231^{53}$

- Domènec Abat

- Mateu Alfeu

- Joan de Banyuls

- Pere Escrivà

- Guillem de l'Estrella

- Guillem dels Horts

- Ramon Mercer

- Guillem de Na Huga

- En Pastor Ninard

- Pere Ninard

- Joan Perés

- Pere Ramon

- Guillem Ros

\section{BIBLIOGRAPHIE CITÉE}

D’Achery, Luc (1723), Spicilegium, rééd. Étienne Baluze et Ed. Edmond Martène, 3 vols., Paris, Montalant.

Aguiló, Estanislao de K. (1912-1915), Capbreu ordenat l'any 1304 dels establiments i donacions fets per Nunó Sanç de la seva porció, "Bolletí de la Societat Arqueológica Lul·liana" 14, pp. 209-224, 241-256, 273-285 et 15, pp. 53-62.

Alart, Bernard (1874), Privilèges et titres relatifs aux franchises, institutions et propriétés communales de Roussillon et de Cerdagne depuis le XI ${ }^{e}$ siècle jusqu'à l'an 1660, İ̀re part, Perpignan, Charles Latrobe.

Alart, Bernard (1881), Documents sur la langue catalane des anciens comtés de Roussillon et de Cerdagne, Paris, Maisonneuve et Cie.

Amari, Michele (1867), Nuovi ricordi arabici su la storia di Genova, "Atti della Società Ligure di Storia Patria” 5, pp. 549-632.

Amari, Michele (1873), Nuovi ricordi arabici su la storia di Genova, Gênes, Imprimerie Royale des Sourds-Muets.

Ayache, Germain (1979), Études d'histoire marocaine, Rabat, Société Marocaine des Éditeurs réunis.

Balan, Pietro (1873) Storia di Gregorio IX e dei suoi tempi, 2 vols., Modène, Tipografia del Commercio.

Balard, Michel (1978), La Romanie génoise (XII ${ }^{e}$-début du XV siècle), 2 vols., Rome, École Française de Rome, 1978.

${ }^{53}$ Germain 1861, vol. I, n X p. 193. 
Basseda, Lluís (1990), Toponymie historique de Catalunya Nord, Prades, CREC - Revista Terra nostra.

Bautier, Robert-Henri (1953-1954), Les relations commerciales entre l'Europe et l'Afrique du Nord et l'équilibre économique méditerranéen du XII ${ }^{e}$ au XIV siècle, "Bulletin philologique et historique du Comité des travaux historiques et scientifiques", pp. 399-416.

Beaumier, Auguste (1860), Roudh el-Kartas: Histoire des souverains du Maghreb (Espagne et Maroc) et annales de la ville de Fès, Paris, Imprimerie impériale.

Belgrano, Luigi Tommaso (1874), Società Ligure di Storia Patria. Anno XVI, "Archivo Storico Italiano" (3e série) 19, pp. 475-497.

Bibolini, Maria (2000), I Libri Iurium della Repubblica di Genova t. I/6, Gênes, Società Ligure di Storia Patria (Fonti per la Storia della Liguria; 13).

Bisson, Thomas N. (1989), Medieval France and her Pyrenean Neighbours: Studies in Early Institutional History, Londres, Bloomsbury Academic.

Bonet, Miguel (1895-1896), Defensa de Mallorca contra Pedro IV de Aragon (1343), "Bolletí de la Societat Arqueológica Lul-liana" 6, pp. 168171, 182-184, 197-198, 216-217 et 262-264.

Bresc, Henri; Doumerc, Bernard; Eddé, Anne-Marie, [et al.] (2001), La Méditerranée entre pays d'Islam et monde latin (milieu $X^{e}$-milieu XIII ${ }^{e}$ siècle): textes et documents, Paris, SEDES.

Bruguera, Jordi (1991), Llibre dels fets del rei en Jaume, 2 vols., Barcelone, Barcino.

Caddeo, Rinaldo; Nani-Mocenigo, Mario Filippo (1942), Storia marittima dell'Italia dall'evo antico ai nostri giorni, t. I, Milan, Garzanti.

Canale, Michelle Giuseppe (1844), Storia civile, commerciale e letteraria dei Genovesi dalle origini all'anno 1797, 4 vols., Gênes, Giovanni Grondona.

Cateura Bennàsser, Pau (1988), La repoblació nobiliaria de Mallorca per Nuno Sans, dans Montpellier, la Couronne d'Aragon et les Pays de Langue d'Oc (1204-1349): Actes du XII Congrès d'histoire de la Couronne d'Aragon, Montpellier 26-29 septembre 1985, Montpellier, Société archéologique de Montpellier, t. II, pp. 99-114.

Chérif, Mohamed (1996), Ceuta aux époques almohade et mérinide, Paris, L'Harmattan.

Claverie, Pierre-Vincent (2013), Les origines génoises de la famille Carròs, "Bibliothèque de l'École des Chartes" 171/2, pp. 537-544.

Deza, Massimiliano (1694), Istoria della famiglia Spinola descritta dalla sue origine fino al secolo XVI, Plaisance, Giovanni Bazachi.

Dini, Bruno (1980), Una pratica di mercatura in formazione (1394-1395), Florence, F. Le Monnier 
Doehaerd, Renée (1941), Les relations commerciales entre Gênes, la Belgique et l'Outremont d'après les archives notariales génoises aux XIII ${ }^{e}$ et $X I V^{e}$ siècles, 3 vols., Bruxelles, Palais des Académies.

Dotson, John E. (2006), Ship Types and Fleet Composition at Genoa and Venice in the Early Thirteenth Century, dans Pryor, John H. (éd.), Logistics of Warfare in the Age of the Crusades, Aldershot, Ashgate, pp. 63-76.

Doubleday, Simon R. (2001), The Lara Family: Crown and Nobility in Medieval Spain (Harvard historical studies, 141), Cambridge, Massachusetts, Harvard University Press.

Dufourcq, Charles-Emmanuel (1955), La question de Ceuta au XIII siècle, "Hespéris" 42, pp. 67-127.

Dufourcq, Charles-Emmanuel (1966), Un projet castillan du XIII ${ }^{e}$ siècle: la croisade de l'Afrique, "Revue d'histoire et de civilisation du Maghreb" 1, pp. 26-51.

Dufourcq, Charles-Emmanuel (1969), L'expansió catalana a la Mediterrània occidental: Segles XIII i XIV, Barcelone, Editorial Vicens-Vives.

Ferhat, Halima (1993), Sabta des origines au XIVìme siècle, Rabat, Éditions Al-Manahil - Ministère des Affaires Culturelles.

Foglietta, Oberto (1585), Historia Genuensium Libri XII, Gênes, Girolamo Bartoli.

Germain, Alexandre (1861), Histoire du commerce de Montpellier, 2 vols., Montpellier, Jean Martel,

Giustiniani, Agostino (1537), Castigatissimi Annali con la loro copiosa tavola della Eccelsca e Illustrissima Republi. di Genoa, Gênes, Antonio Bellono.

Heyd, Wilhelm (1866-1868), Le colonie commerciali degli Italiani in Oriente nel medio evo, 2 vols., Venise - Turin, Antonelli \& Basadonna.

Huici Miranda, Ambrosio (1964), Rawd el-Qirtas, 2 vols., Valence, Anubar.

Imperiale di Sant'Angelo, Cesare (1923), Annali Genovesi di Caffaro e de' suoi continuatori dal MCCXXV al MCCL, Nuova edizione, t. III, Rome, Istituto Storico Italiano (Fonti per la Storia d'Italia; 13).

Jehel, Georges (1993), Les Génois en Méditerranée occidentale (fin XI'me-début XIV ème siècle): Ébauche d'une stratégie pour un empire, Amiens, Centre d'histoire des sociétés, Université de Picardie.

Jehel, Georges, Racinet, Philippe (2000), Les relations des pays d'Islam avec le monde latin, Paris, Éditions du Temps.

Jehel, Georges (2001), L'Italie et le Maghreb au Moyen Âge: Conflits et échanges du VII ème au XV $V^{\text {ème }}$ siècle, Paris, Presses Universitaires de France.

Jehel, Georges (2015), La place de Ceuta dans le réseau portuaire euro-méditerranéen $\left(X I I^{e}-X V^{e}\right.$ siècles $)$, dans Cancellieri, Jean-André; Marchi 
van Cauwelaert, Vannina (éd.), Villes portuaires de Méditerranée occidentale au Moyen Âge. Îles et continents, XII ${ }^{e}-X V^{e}$ siècles, Palerme, Associazione Mediterranea, pp. 57-76 (Quaderni Mediterranea ricerche storiche; 26).

Lalinde Abadía, Jesús (1979), La Corona de Aragón en el Mediterráneo medieval, 1229-1479, Saragosse, Institución Fernando el Católico, CSIC.

Mas Latrie, Louis de (1866), Traités de paix et de commerce et documents divers concernant les relations des chrétiens avec les Arabes de l'Afrique septentrionale au Moyen Age, Paris, Plon.

Matthieu Paris (1876), Chronica Majora, Luard, Henry Richards (éd.), t. III, Londres, Longman \& Co.

Miret i Sans, Joaquim (2007), Itinerari de Jaume el Conqueridor ( $2^{\mathrm{e}}$ éd. en facsimilé) Barcelone, Institut d'Estudis Catalans (Memòries de la Secció Històrico-Arqueològica; 65).

Misbach, Henry Lorenz (1968), Genoese Trade and the Flow of Gold, 1154-1253, Ann Arbor, Université du Wisconsin (thèse dactylographiée).

Moran i Ocerinjauregui, Josep (1998), Cronicó de Perpinyà (segle XIII). Estudi filològic $i$ lingüístic, Montserrat, Publicacions de l'Abadia de Montserrat.

Mosquera Merino, María del Carmen (1991), Ceuta en el siglo XIII, 2 vols., Madrid, Universidad Complutense (thèse dactylographiée).

Mosquera Merino, María del Carmen (1994), La señoría de Ceuta en el siglo XIII: historia política y económica, Ceuta, Instituto de Estudios Ceutíes.

Mosquera Merino, María del Carmen (2002), La Señoría de Ceuta y el dominio sobre el Estrecho de Gibraltar, dans Ceuta en el Medievo: la ciudad en el universo árabe, Ceuta, Instituto de Estudios Ceutíes, pp. 109-130

Pegolotti, Francesco Balducci (1936), La pratica della mercatura, ed. Allan Evans, Cambridge, Massachusetts (The Mediaeval Academy of America Publication; 24).

Pérez, Lorenzo (1961-1967), Documentos conservados en los registros Vaticanos relativos al primer pontificado de Mallorca (12301266), "Bolletí de la Societat Arqueológica Lul·liana" 32, pp. 48-66.

Picard, Christophe (1997), L'océan Atlantique musulman de la conquête arabe à l'époque almohade, Paris, Maisonneuve et Larose.

Picard, Christophe (2015), La Mer des Califes: Une histoire de la Méditerranée musulmane (VII ${ }^{e}$ XII ${ }^{e}$ siècle), Paris, Le Seuil. 
Pistarino, Geo (1980), Genova e l'Islam nel Mediterraneo occidentale, s. XIIXIII, "Anuario de Estudios Medievales" 10, pp. 189-205.

Promis, Domenico (1865), La Zecca di Scio durante il Dominio dei Genove$s i$, Turin, Stamperia Reale.

Puncuh, Dino (1996), I Libri Iurium della Repubblica di Genova, t. I-2, Gênes, Società Ligure di Storia Patria (Fonti per la Storia della Liguria; 13).

Refass, Mohamed (1996), L'organisation de la péninsule tingitane, Rabat, Université Mohammed V, Publication de la Faculté des Lettres et des Sciences Humaines.

Rosenberger, Pierre (1995), Le contrôle du détroit de Gibraltar aux XII et XIII ${ }^{e}$ siècles, dans M. Hammam, Mohammed (éd.), L'Occident musulman et l'Occident chrétien au Moyen Âge, Rabat, Université Mohammed V, Publication de la Faculté des Lettres et des Sciences Humaines, pp. 15-42.

Santamaría, Álvaro (1990), Ejecutoria del Reino de Mallorca, Palma de Majorque, Ajuntament de Palma.

Teulet, Alexandre (1866), Layettes du Trésor des Chartes, t. II, Paris, Imprimerie impériale.

Tréton, Rodrigue; Catafau, Aymat; Verdon, Laure (2011), Les capbreus $d u$ roi Jacques II de Majorque (1292-1294), 2 vols., Paris, Éditions du Comité des travaux historiques et scientifiques.

Tréton, Rodrigue; Vinas, Robert (2017), Le testament de Nunó Sanç, seigneur de Roussillon et de Cerdagne (17 décembre 1241), "e-Spania" 28 (https://e-spania.revues.org/27026) [consulté: 9/02/2018].

Tucci, Raffaele Di (1935), Documenti inediti sulla spedizione e sulla mahona dei Genovesi a Ceuta (1234-1237), “Atti della Società Ligure di Storia Patria" 64, pp. 271-340.

Udina i Abelló, Antoni (2001), Els testaments dels comtes de Barcelona i dels reis de la Corona d'Aragó: De Guifré Borrell a Joan II, Barcelone, Fundació Noguera.

Unali, Anna (2000), Ceuta 1415: alle origini dell'espansione europea in Africa, Rome, Bulzoni Editore.

Valérian, Dominique (2006), Bougie port maghrébin, 1067-1510, Rome, École française de Rome (BEFAR; 328).

Vidal, Pierre (1887), Documents sur la langue catalane des anciens comtés de Roussillon et de Cerdagne (1311-1380), "Revue des Langues Romanes" 31, pp. 59-78.

Vich y Salom, Juan; Muntaner y Bujosa, Juan (1945), Documenta regni Majoricarum (Miscelanea), Palma de Majorque, Imprenta Amengual y Muntaner. 
Villanueva, Jaime (1851), Viage á Mallorca, "Viage literario a las iglesias de España" 21,pp. 1-321.

Zaïm, Fouad (1990), Le Maroc et son espace méditerranéen: histoire économique et sociale, Rabat, Confluences.

Fecha de recepción del artículo: agosto 2015

Fecha de aceptación y versión final: junio 2016 